\title{
Flavour Mixing of Neutrinos and Baryon Asymmetry of the Universe
}

\author{
Takehiko Asaka ${ }^{1}$ and Hiroyuki Ishida ${ }^{2}$ \\ ${ }^{1}$ Department of Physics, Niigata University, Niigata 950-2181, Japan \\ ${ }^{2}$ Graduate School of Science and Technology, Niigata University, Niigata 950-2181, Japan
}

(April 30, 2010)

\begin{abstract}
We investigate baryogenesis in the $\nu$ MSM, which is the Minimal Standard Model (MSM) extended by three right-handed neutrinos with Majorana masses smaller than the weak scale. In this model the baryon asymmetry of the universe (BAU) is generated via flavour oscillation between right-handed neutrinos. We consider the case when BAU is solely originated from the $\mathrm{CP}$ violation in the mixing matrix of active neutrinos. We perform analytical and numerical estimations of the yield of BAU, and show how BAU depends on mixing angles and $\mathrm{CP}$ violating phases. It is found that the asymmetry in the inverted hierarchy for neutrino masses receives a suppression factor of about $4 \%$ comparing with the normal hierarchy case. It is, however, pointed out that, when $\theta_{13}=0$ and $\theta_{23}=\pi / 4$, baryogenesis in the normal hierarchy becomes ineffective, and hence the inverted hierarchy case becomes significant to account for the present BAU.
\end{abstract}




\section{Introduction}

The origin of the baryon asymmetry of the universe (BAU) is one of the most mysterious problems in particle physics and cosmology, since the Minimal Standard Model (MSM) and the Big Bang cosmology cannot answer it. So far various mechanisms for generating BAU have been proposed [1]. One promising possibility is the so-called leptogenesis scenario [2] (see also Ref. [3]), where the origins of neutrino masses as well as BAU can be addressed at the same time by introducing right-handed neutrinos with superheavy Majorana masses. The observed tiny masses of neutrinos can be naturally understood by the seesaw mechanism [4]. Further, the lepton asymmetry generated by decays of right-handed neutrinos can be a source of BAU. In the simplest thermal leptogenesis, the required Majorana masses is heavier than about $10^{9}$ $\mathrm{GeV}[5]$.

It should be, however, noted that the connection between the origins of neutrino masses and BAU can be obtained even when Majorana masses are below the weak scale. One interesting possibility is the so-called $\nu$ MSM [6, 7], which is the MSM extended by three right-handed neutrinos with masses smaller than the weak scale. In this model the problems of neutrino masses, BAU and also dark matter can be solved simultaneously. One attractive advantage of the $\nu$ MSM lies in the fact that the direct detection of right-handed neutrinos becomes possible [8].

In the $\nu$ MSM BAU can be generated by invoking the mechanism via flavour oscillation of right-handed neutrinos [9]. (See also Ref. [6, 10].) In this mechanism the lepton asymmetry is not generated for temperatures of interest because of the smallness of Majorana masses, which is very different from the leptogenesis scenario. The lepton asymmetry is separated into left-handed and right-handed leptonic sectors due to the CP violations in the production and oscillation of right-handed neutrinos. Then, the asymmetry stored in the left-handed sector is partially transferred into the baryon asymmetry through the rapid sphaleron transitions [11].

One of right-handed neutrinos in the $\nu \mathrm{MSM}$, which is a candidate of dark matter, plays no essential role to generate BAU as well as neutrino masses observed in the oscillation experiments, since its Yukawa coupling constants should be very suppressed. The rest two are responsible to BAU via their flavour oscillation, but also induce the masses of active neutrinos through the seesaw mechanism. Therefore, physics of these two right-handed neutrinos connects BAU with the neutrino parameters of active neutrinos, i.e., mass hierarchy, mixing angles, and CP violating phases.

In this letter we would like to extend the analysis in Ref. [6]. Under the considering situation there are three CP violating phases in the leptonic sector which can be a source of the asymmetry. Especially, we concentrate here on the case when BAU is originated only from the $\mathrm{CP}$ violation in the mixing matrix $U$ of active neutrinos, namely, the Dirac phase $\delta$ and Majorana phase $\eta$ in $U$. We then present the analytical expression of BAU shows explicitly how BAU depends on these CP phases and the mixing angles of active neutrinos. Moreover, we also 
perform the numerical estimation of BAU and justify the validity of the analytical expression.

\section{The $\nu \mathrm{MSM}$}

We begin with the brief review of the model under consideration, i.e., the $\nu$ MSM [6, 7]. It is the MSM extended by three right-handed neutrinos $\nu_{R I}(I=1,2,3)$, which Lagrangian is given by

$$
\mathcal{L}_{\nu \mathrm{MSM}}=\mathcal{L}_{\mathrm{MSM}}+i \overline{\nu_{R I}} \gamma^{\mu} \partial_{\mu} \nu_{R I}-\left(F_{\alpha I} \bar{L}_{\alpha} \Phi \nu_{R I}+\frac{M_{I}}{2} \overline{\nu_{R}^{c}} \nu_{R I}+\text { h.c. }\right),
$$

where $\mathcal{L}_{\text {MSM }}$ is the MSM Lagrangian. $F_{\alpha I}$ are neutrino Yukawa couplings, and $\Phi$ and $L_{\alpha}$ $(\alpha=e, \mu, \tau)$ are Higgs and lepton weak-doublets, respectively. The Majorana masses of righthanded neutrinos are denoted by $M_{I}$ which are taken to be real and positive without loss of generality. Here and hereafter we work in a basis in which the mass matrix of charged leptons is diagonal. In this model, neutrinos also obtain the Dirac masses, $\left[M_{D}\right]_{\alpha I}=F_{\alpha I}\langle\Phi\rangle(\langle\Phi\rangle$ is a vacuum expectation value of the Higgs field), after the electroweak (EW) symmetry breaking.

The distinctive feature of the model is the region of the parameter space of Eq. (1), i.e., we restrict ourselves in the region

$$
\left|\left[M_{D}\right]_{\alpha I}\right| \ll M_{I} \lesssim 100 \mathrm{GeV}
$$

In this case the seesaw mechanism works, and mass eigenstates of neutrinos are then divided into two groups. One group consists of active neutrinos $\nu_{i}(i=1,2,3)$. Their masses are found from the seesaw mass matrix $M_{\nu}=-M_{D} M_{I}^{-1} M_{D}^{T}$ as

$$
U^{\dagger} M_{\nu} U^{*}=\operatorname{diag}\left(m_{1}, m_{2}, m_{3}\right)
$$

where $U$ is the mixing matrix of active neutrinos. The other one consists of sterile neutrinos $N_{I}$ which are almost the right-handed states $N_{I} \simeq \nu_{R I}$, and their masses are approximately given by the Majorana masses $M_{I}$. We then find the neutrino mixing as

$$
\nu_{L \alpha}=U_{\alpha i} \nu_{i}+\Theta_{\alpha I} N_{I}^{c}
$$

where $\Theta_{\alpha I}=\left[M_{D}\right]_{\alpha I} / M_{I}$ are the active-sterile (left-right) mixing matrix. We should stress that sterile neutrinos $N_{I}$ here are originated from the right-handed neutrinos in the seesaw mechanism. Thus, we simply say $N_{I}$ as right-handed neutrinos from now on.

In the $\nu \mathrm{MSM}$ three right-handed neutrinos play important roles in cosmology. One of them, say $N_{1}$, is a candidate for dark matter of the universe [7]. It is beyond the scope of the present work to describe this issue. However, one thing being important for later discussions is that the Yukawa couplings of $N_{1}$ should be highly suppressed to realise a successful dark matter scenario. (See the details, e.g., Refs. [12, 10, 13, 14].) As a result, the contribution from $N_{1}$ to the seesaw matrix $M_{\nu}$ becomes negligible [7]. Furthermore, as shown in Ref. [6], $N_{1}$ plays 
essentially no role to generate BAU. Therefore, we take $F_{\alpha 1}=0$ for simplicity in the rest of this analysis.

The other right-handed neutrinos, $N_{2}$ and $N_{3}$, are then responsible to the masses and mixing angles of active neutrinos. Notice that the lightest active neutrino becomes massless in our approximation. Further, the flavour oscillation between $N_{2}$ and $N_{3}$ in the early universe can be a source of BAU through the mechanism proposed in Ref. [9], as we will show below. In the $\nu \mathrm{MSM}$, therefore, BAU is related to the parameters of active neutrinos through physics of $N_{2}$ and $N_{3}$.

The neutrino Yukawa matrix $F$ for $N_{2}$ and $N_{3}$, which is a $3 \times 2$ matrix, can be expressed without loss of generality as [15]

$$
F=\frac{i}{\langle\Phi\rangle} U D_{\nu}^{1 / 2} \Omega D_{N}^{1 / 2}
$$

Here parameters of active neutrinos are their masses $D_{\nu}=\operatorname{diag}\left(m_{1}, m_{2}, m_{3}\right)$ and the mixing matrix

$$
U=\left(\begin{array}{ccc}
c_{12} c_{13} & s_{12} c_{13} & s_{13} e^{-i \delta} \\
-c_{23} s_{12}-s_{23} c_{12} s_{13} e^{i \delta} & c_{23} c_{12}-s_{23} s_{12} s_{13} e^{i \delta} & s_{23} c_{13} \\
s_{23} s_{12}-c_{23} c_{12} s_{13} e^{i \delta} & -s_{23} c_{12}-c_{23} s_{12} s_{13} e^{i \delta} & c_{23} c_{13}
\end{array}\right) \times \operatorname{diag}\left(1, e^{i \eta}, 1\right),
$$

with $s_{i j}=\sin \theta_{i j}$ and $c_{i j}=\cos \theta_{i j}$. The Dirac and Majorana phases are denoted by $\delta$ and $\eta$, respectively. Since we set $F_{\alpha 1}=0$, the masses of active neutrinos are

$$
\begin{aligned}
& m_{3}=m_{\mathrm{atm}}>m_{2}=m_{\mathrm{sol}}>m_{1}=0 \text { in the NH case } \\
& m_{2}=\sqrt{m_{\mathrm{atm}}^{2}+m_{\mathrm{sol}}^{2}}>m_{1}=\sqrt{m_{\mathrm{atm}}^{2}}>m_{3}=0 \text { in the IH case, }
\end{aligned}
$$

The observational data of mixing angles are $s_{12}^{2}=0.318_{-0.048}^{+0.062}, s_{23}^{2}=0.50_{-0.14}^{+0.17}$, and $s_{13}^{2} \leq 0.053$, respectively, and masses are $m_{\text {sol }}^{2}=\Delta m_{21}^{2}=\left(7.59_{-0.56}^{+0.68}\right) \times 10^{-5} \mathrm{eV}^{2}$, and $m_{\text {atm }}^{2}=\left|\Delta m_{31}^{2}\right|=$ $\left(2.40_{-0.33}^{+0.35}\right) \times 10^{-3} \mathrm{eV}^{2}$ (at the $3 \sigma$ level) [16]. Hereafter, we shall adopt the central values unless otherwise stated.

On the other hand, parameters of $N_{2}$ and $N_{3}$ are their masses $D_{N}=\operatorname{diag}\left(M_{2}, M_{3}\right)$ and the $3 \times 2$ matrix

$$
\begin{aligned}
& \Omega=\left(\begin{array}{cc}
0 & 0 \\
\cos \omega & -\sin \omega \\
\xi \sin \omega & \xi \cos \omega
\end{array}\right) \text { in the NH case, } \\
& \Omega=\left(\begin{array}{cc}
\cos \omega & -\sin \omega \\
\xi \sin \omega & \xi \cos \omega \\
0 & 0
\end{array}\right) \text { in the IH case, }
\end{aligned}
$$

where $\xi= \pm 1$ and $\omega$ is an arbitrary complex number.

In the considering situation there are three $\mathrm{CP}$ violating parameters, $\delta, \eta$ and $\operatorname{Im} \omega$, in leptonic sector, which can potentially contribute to the generation of BAU. In this analysis we 
concentrate on the case in which BAU is originated solely from the CP phases in the mixing matrix of active neutrinos, and find the dependence on $\delta$ and $\eta$ as well as the mixing angles $\theta_{i j}$ by taking $\operatorname{Im} \omega=0$

\section{Baryogenesis via Neutrino Oscillation}

Let us then discuss how BAU is generated in the $\nu \mathrm{MSM}$ through baryogenesis via oscillation of right-handed neutrinos [9]. In the considering model the lepton-number violations due to Majorana masses are ineffective for high temperatures $T \gtrsim 100 \mathrm{GeV}[9]$. In order to generate the baryon asymmetry, thus, it is crucial the lepton asymmetry is distributed into left-handed leptons $L_{\alpha}$ and right-handed neutrinos $N_{I}$ for the temperatures of interest rather than its creation. Then, the asymmetry stored in the left-handed sector is partially transferred into the baryon asymmetry due to the $B+L$ breaking sphaleron transition which is rapid for $T>T_{W} \simeq 100 \mathrm{GeV}[11]$.

We denote the asymmetries of numbers of $N_{2,3}$ and $L_{\alpha}$ by $\Delta N_{2,3}$ and $\Delta L_{\alpha}$, respectively. These asymmetries are estimated by solving the kinetic equations for their density matrices $\rho_{N N}$ and $\rho_{L I} \# 2$, which are given by

$$
\begin{aligned}
i \frac{d \rho_{N N}}{d t} & =\left[H_{N N}^{0}+V_{N}, \rho_{N N}\right]-\frac{i}{2}\left\{\Gamma_{N}, \rho_{N N}-\rho_{N N}^{e q}\right\}+\frac{i \sin \phi}{8} T F^{\dagger}\left(\rho_{L L}-\rho_{L L}^{e q}\right) F, \\
i \frac{d \rho_{L L}}{d t} & =\left[H_{L L}^{0}+V_{L}, \rho_{L L}\right]-\frac{i}{2}\left\{\Gamma_{L}, \rho_{L L}-\rho_{L L}^{e q}\right\}+\frac{i \sin \phi}{4} T F\left(\rho_{N N}-\rho_{N N}^{e q}\right) F^{\dagger},
\end{aligned}
$$

where note again that $F$ is the $3 \times 2$ matrix for neutrino Yukawa couplings of $N_{2}$ and $N_{3}$. $H_{N N}^{0}$ and $H_{L L}^{0}$ denote the Hamiltonian when $F=0$. The effective potentials and the destruction rates for $N$ and $L_{\alpha}$ are

$$
\begin{array}{rlrl}
V_{N} & =\frac{1}{8} T F^{\dagger} F, & \Gamma_{N}=2 s_{\phi} V_{N} \\
V_{L}=\frac{1}{16} T F^{\dagger} F, & \Gamma_{L}=2 s_{\phi} V_{L} .
\end{array}
$$

where $s_{\phi} \simeq 2 \times 10^{-2}[9]$. Notice that these expressions are valid as long as $T$ is sufficiently higher than $T_{W}$. The kinetic equations of the density matrices for the anti-particles $\rho_{\bar{N}} \bar{N}$ and $\rho_{\bar{L} \bar{L}}$ are obtained by replacing $F \rightarrow F^{*}$ in Eqs. (9) and (10).

These equations include the medium effects of surrounding hot plasma, i.e., the thermal potentials which describe the coherent oscillations of right-handed neutrinos induced by $V_{N}$, and the decoherent terms which describe the production and destruction of $N_{I}$ [9]. Furthermore, we include the terms which express the exchange of asymmetries between left and right-handed sectors [6]. It is found from Eqs. (9) and (10) that $\Delta N_{\text {tot }}+\Delta L_{\text {tot }}=0$, where $\Delta N_{\text {tot }}=\sum_{I=2,3} \Delta N_{I}$

\footnotetext{
\#1 The impact of Im $\omega$ on BAU will be discussed in elsewhere [17.

\#2 The density matrix $\rho_{L L}$ denotes the sum of $\rho_{\nu_{L} \nu_{L}}$ and $\rho_{e_{L} e_{L}}$, which are the same in the temperatures under consideration due to $\mathrm{SU}(2)_{L}$ symmetry.
} 
and $\Delta L_{\text {tot }}=\sum_{\alpha=e, \mu, \tau} \Delta L_{\alpha}$, which is crucial in the considering baryogenesis scenario as mentioned above. For further details of these issues, please see Ref. [6].

The coupled equations (9) and (10) can be solved not only numerically, but also analytically by using the perturbative expansion of the Yukawa coupling constants $F$ [6]. The initial conditions are taken as $\rho_{N N}(0)=\rho_{\bar{N} \bar{N}}(0)=0$ and $\rho_{L L}(0)=\rho_{\bar{L} \bar{L}}(0)=\rho_{L L}^{e q}$. Then, we can estimate the asymmetries as $\Delta N_{I}=\left[\rho_{N N}\right]_{I I}-\left[\rho_{\bar{N} \bar{N}}\right]_{I I}$ and $\Delta L_{\alpha}=\left[\rho_{L L}\right]_{\alpha \alpha}-\left[\rho_{\bar{L} \bar{L}}\right]_{\alpha \alpha}$. In the following we will present the analytical expression of the active flavour asymmetry as well as BAU at the leading order of $F$. We will also show the results from the numerical solutions of Eqs. (9) and (10), which confirm the validity of the analytical expressions of the asymmetries.

\section{Active Flavour Asymmetries}

First of all, we discuss the yield of the active flavour asymmetry $\Delta L_{\alpha}(\alpha=e, \mu, \tau)$. The leading order contribution to $\Delta L_{\alpha}$ is induced at $\mathcal{O}\left(F^{4}\right)$ and the analytic expression at the temperature $T$ can be written as 6 ]

$$
\Delta L_{\alpha}(T)=\frac{s_{\phi}^{2}}{4} A_{32}^{\alpha} \frac{M_{0}^{2}}{T_{L}^{2}} J_{32}\left(T_{L} / T\right),
$$

where $M_{0} \simeq 7.1 \times 10^{17} \mathrm{GeV}$ and the $\mathrm{CP}$ asymmetry parameter $A_{32}^{\alpha}$ is defined by

$$
A_{32}^{\alpha}=\operatorname{Im}\left[F_{\alpha 3}\left[F^{\dagger} F\right]_{32} F_{\alpha 2}^{*}\right] .
$$

The typical temperature of the generation of $\Delta L_{\alpha}, T_{L}$, is given by

$$
T_{L}=\left(\frac{1}{6} M_{0} \Delta M_{32}^{2}\right)^{\frac{1}{3}}=2.2 \mathrm{TeV}\left(\frac{M_{3}}{3 \mathrm{GeV}}\right)^{\frac{2}{3}}\left(\frac{\Delta M_{32}^{2} / M_{3}^{2}}{10^{-8}}\right)^{\frac{1}{3}}
$$

where $\Delta M_{32}^{2}=M_{3}^{2}-M_{2}^{2}$. The evolution of $\Delta L_{\alpha}$ is described by the function $J_{32}$

$$
J_{32}(x)=\int_{0}^{x} d x_{1} \int_{0}^{x_{1}} d x_{2} \sin \left(x_{1}^{3}-x_{2}^{3}\right) .
$$

It is found that $J_{32}(x) \simeq \frac{3}{20} x^{5}$ for $x \ll 1$ while it is oscillating around and approaching to the value

$$
\left.J_{32}(x)\right|_{x \gg 1}=\frac{2^{1 / 3} \pi^{3 / 2}}{9 \Gamma(5 / 6)}=0.69
$$

Therefore, the active flavour asymmetry scales as $\Delta L_{\alpha} \propto T^{5}$ for $T \gg T_{L}$ and takes a constant value for $T \ll T_{L}$ as

$$
\Delta L_{\alpha}=\frac{3^{2 / 3} \pi^{3 / 2} s_{\phi}^{2}}{18 \Gamma(5 / 6)} A_{32}^{\alpha} \frac{M_{0}^{4 / 3}}{\left(\Delta M_{32}^{2}\right)^{2 / 3}} .
$$

We can see that $\Delta L_{\alpha}$ for $T \ll T_{L}$ is enhanced when $N_{2}$ and $N_{3}$ are quasi degenerate [6]. 
Now we would like to express the CP asymmetry parameter $A_{32}^{\alpha}$ by using the parameters of the neutrino Yukawa couplings in Eq. (5) . In the $\mathrm{NH}$ case we evaluate $A_{32}^{\alpha}$ as

$$
\begin{aligned}
A_{32}^{\alpha} & =\xi \sin 2 \operatorname{Re} \omega \frac{M_{3} M_{2}\left(m_{3} m_{2}\right)^{1 / 2}\left(m_{3}-m_{2}\right)}{4\langle\Phi\rangle^{4}} \times a_{\alpha} \\
& =\xi \sin 2 \operatorname{Re} \omega \frac{M_{3} M_{2} m_{\mathrm{atm}}^{3 / 2} m_{\mathrm{sol}}^{1 / 2}}{4\langle\Phi\rangle^{4}} \times S_{m_{\nu}} \times a_{\alpha},
\end{aligned}
$$

where $S_{m_{\nu}}$ is

$$
S_{m_{\nu}}=1-r_{m}
$$

where $r_{m}=m_{\mathrm{sol}} / m_{\mathrm{atm}}$. The parameter $a_{\alpha}$ can be evaluated as

$$
\begin{aligned}
& a_{e}=-\sin \theta_{12} \sin 2 \theta_{13} \sin (\delta+\eta), \\
& a_{\mu}=+\sin ^{2} \theta_{23} \sin \theta_{12} \sin 2 \theta_{13} \sin (\delta+\eta)-\sin 2 \theta_{23} \cos \theta_{12} \cos \theta_{13} \sin \eta \\
& a_{\tau}=+\cos ^{2} \theta_{23} \sin \theta_{12} \sin 2 \theta_{13} \sin (\delta+\eta)+\sin 2 \theta_{23} \cos \theta_{12} \cos \theta_{13} \sin \eta
\end{aligned}
$$

We find that the active flavour asymmetry depends on parameters of right-handed neutrinos as $\Delta L_{\alpha} \propto \xi \sin 2 \operatorname{Re} \omega M_{2} M_{3} /\left(\Delta M_{32}^{2}\right)^{2 / 3}$, and hence vanishes when Rew $=n \pi / 2$ ( $n$ is integer). This is simply because the flavour oscillation between $N_{2}$ and $N_{3}$, which is essential to the considering mechanism, disappears. On the other hand, the dependence on the mixing angles and $\mathrm{CP}$ violating phases of active neutrinos is summarised in $a_{\alpha}$.

As already pointed out in Ref. [6], the total asymmetry of active flavours vanishes $\Delta L_{\text {tot }}=0$ at the leading $\mathcal{O}\left(F^{4}\right)$ since $\sum_{\alpha} A_{32}^{\alpha}=\operatorname{Im}\left[\left[F^{\dagger} F\right]_{23}\left[F^{\dagger} F\right]_{32}\right]=0$, which can be seen as $a_{e}+a_{\mu}+$ $a_{\tau}=0$ in Eq. (21). It is interesting to note that the active flavour asymmetries depend on CP violating phases in two ways, i.e., $\sin (\delta+\eta)$ and $\sin \eta$, and $\Delta L_{e}$ only depends on the former one. We also find that $\Delta L_{e}$ vanishes and $\Delta L_{\mu}=-\Delta L_{\tau}$ when $\theta_{13}=0$. In this case $a_{e}=0$ and $a_{\mu}=-a_{\tau}=-\sin 2 \theta_{23} \cos \theta_{12} \sin \eta$, and the asymmetries only depend on Majorana phase $\eta$ as expected.

On the other hand, in the inverted hierarchy case, the CP asymmetry parameter $A_{32}^{\alpha}$ can be written as

$$
\begin{aligned}
A_{32}^{\alpha} & =\xi \sin 2 \operatorname{Re} \omega \frac{M_{3} M_{2}\left(m_{2} m_{1}\right)^{1 / 2}\left(m_{2}-m_{1}\right)}{4\langle\Phi\rangle^{4}} \times a_{\alpha} \\
& =\xi \sin 2 \operatorname{Re} \omega \frac{M_{3} M_{2} m_{\mathrm{atm}}^{3 / 2} m_{\mathrm{sol}}^{1 / 2}}{4\langle\Phi\rangle^{4}} \times S_{m_{\nu}} \times a_{\alpha},
\end{aligned}
$$


where $a_{\alpha}$ is found as

$$
\begin{aligned}
a_{e}= & +\sin 2 \theta_{12} \cos ^{2} \theta_{13} \sin \eta \\
a_{\mu}= & -\left[\sin ^{2} \theta_{23} \cos ^{2} \theta_{13}+\cos 2 \theta_{23}\right] \sin 2 \theta_{12} \sin \eta \\
& +\sin 2 \theta_{23} \sin \theta_{13}\left[\cos \eta \sin \delta-\cos 2 \theta_{12} \sin \eta \cos \delta\right], \\
a_{\tau}= & -\left[\cos ^{2} \theta_{23} \cos ^{2} \theta_{13}-\cos 2 \theta_{23}\right] \sin 2 \theta_{12} \sin \eta \\
& -\sin 2 \theta_{23} \sin \theta_{13}\left[\cos \eta \sin \delta-\cos 2 \theta_{12} \sin \eta \cos \delta\right] .
\end{aligned}
$$

Further, we have introduced

$$
S_{m_{\nu}}=r_{m}^{-\frac{1}{2}}\left(1+r_{m}^{2}\right)^{\frac{1}{4}}\left[\left(1+r_{m}^{2}\right)^{\frac{1}{2}}-1\right]=\frac{r_{m}^{\frac{3}{2}}}{2}\left[1-\frac{r_{m}^{4}}{32}+\mathcal{O}\left(r_{m}^{6}\right)\right]
$$

We find that the active flavour asymmetry in the IH depends on the parameters of righthanded neutrinos in the same way as in the NH. The total asymmetry of active flavours at $\mathcal{O}\left(F^{4}\right)$ vanishes $\Delta L_{\text {tot }}=0$ as in the $\mathrm{NH}$ case.

It should be noted that, comparing with the NH case (19), $A_{32}^{\alpha}$ in the IH case receives of suppression factor of $S_{m_{\nu}} \simeq 0.04$, and thus the production of active flavour asymmetries in $\mathrm{IH}$ is less effective than NH apart from $a_{\alpha}$. Further, $\Delta L_{\alpha}$ depends on CP violating phases differently from the NH case. When $\theta_{13}=0$, we find that

$$
\begin{aligned}
& a_{e}=+\sin 2 \theta_{12} \sin \eta, \\
& a_{\mu}=-\sin 2 \theta_{12} \cos ^{2} \theta_{23} \sin \eta, \\
& a_{\tau}=-\sin 2 \theta_{12} \sin ^{2} \theta_{23} \sin \eta .
\end{aligned}
$$

Thus, as in the NH case, the asymmetries only depend on Majorana phase $\eta$, as expected. In this case, however, $\Delta L_{e}$ does not vanish even when $\theta_{13}=0$, and we have $\Delta L_{\mu}=\Delta L_{\tau}=-0.5 \Delta L_{e}$ for $\theta_{23}=\pi / 4$.

The total asymmetry $\Delta L_{\text {tot }}$ is induced at $\mathcal{O}\left(F^{6}\right)$ and $\Delta L_{\text {tot }}=-\Delta N_{\text {tot }}$. It is found in Ref. [6] that

$$
\begin{aligned}
\Delta L_{\mathrm{tot}}(T) & =-\frac{s_{\phi}}{8} \int_{T}^{\infty} d T_{1} \frac{M_{0}}{T_{1}^{2}} \sum_{I=2,3} \sum_{\alpha=e, \mu, \tau}\left|F_{I \alpha}^{2}\right| \Delta L_{\alpha}(T) \\
& =-\frac{s_{\phi}^{3}}{32} \sum_{I=2,3} \sum_{\alpha=e, \mu, \tau}\left|F_{I \alpha}^{2}\right| A_{32}^{\alpha} \frac{M_{0}^{3}}{T_{L}^{3}} K\left(T_{L} / T\right),
\end{aligned}
$$

where the evolution of $\Delta L_{\text {tot }}$ is described by the function $K$ :

$$
K(x)=\int_{0}^{x} d x_{1} J_{32}\left(x_{1}\right) .
$$

We find that $K(x) \simeq x^{6} / 40$ for $x \ll 1$ while $K(x) \simeq J_{32}(\infty) x$ for $x \gg 1 \# 3$ Then, we normalise

${ }^{\# 3}$ We numerically find that $K(x)$ can be fitted as $K(x)=a x-b$ for $2 \lesssim x \lesssim 100$, where $a=0.68 \simeq J_{32}(\infty)$ and $b=1.4$. 


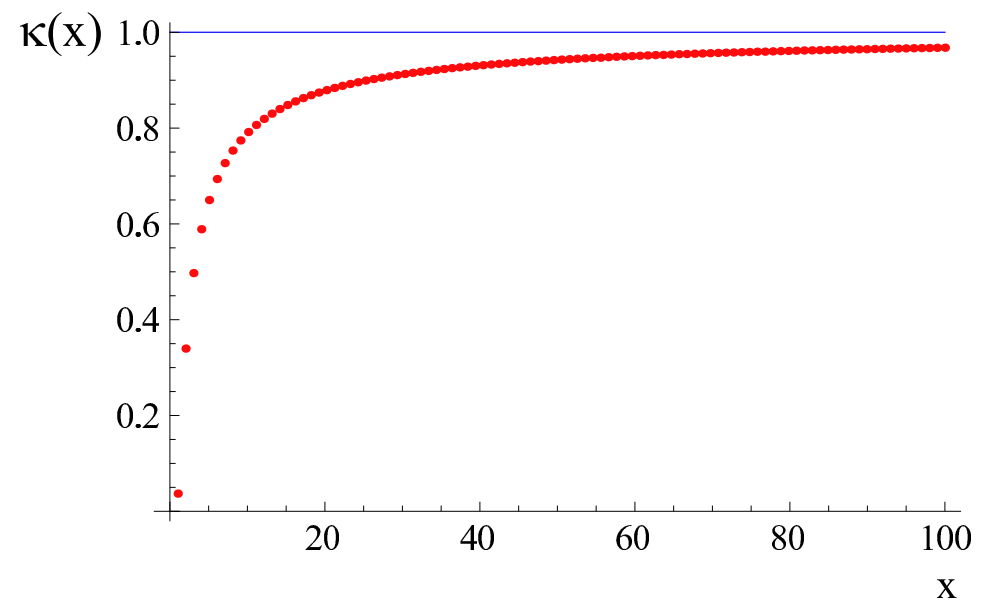

Figure 1: Function $\kappa(x)$ in Eq. (28).

$K(x)$ as

$$
K(x)=J_{32}(\infty) x \kappa(x),
$$

where the behaviour of the function $\kappa(x)$ is shown in Fig. 1.

It should be noted that we have so far assumed that $N_{2}$ and $N_{3}$ are out of equilibrium in order to avoid the wash-out of the asymmetries. When the interaction rate of right-handed neutrinos (11) is $\Gamma_{N}<H$ (the Hubble parameter) till the sphaleron transitions are switched off at $T=T_{W} \simeq 100 \mathrm{GeV}$, the eigenvalues of $F^{\dagger} F$ should be smaller than $2.8 \times 10^{-14}$. This is translated into the upper bound on the mass of right-handed neutrinos as $M_{2,3} \lesssim 17 \mathrm{GeV}[6]$ for both the $\mathrm{NH}$ and IH cases by taking $N_{2}$ and $N_{3}$ are quasi-degenerate, which is required to explain the observed BAU as we will show. We thus restrict ourselves in this mass region below.

\section{Baryon Asymmetry of the Universe}

Now we are at the position to present the analytical expression of BAU and study how it depends on masses, mixing angles and $\mathrm{CP}$ violating phases of active neutrinos. The baryonto-entropy ratio, $Y_{B}=n_{B} / s\left(n_{B}\right.$ and $s$ are the baryon and entropy densities at the present universe, respectively), is obtained from $\Delta L_{\text {tot }}$ as

$$
Y_{B}=-c_{s} \frac{28}{79} \Delta L_{\text {tot }}\left(T_{W}\right),
$$

where $c_{s}=7.0 \times 10^{-4}$. Notice that only the asymmetry in the left-handed leptons is transferred via the sphaleron processes into the baryon asymmetry as $\Delta B=-\frac{28}{79} \Delta L_{\text {tot }}$ [18], and this transition is switched off for $T<T_{W}$.

We take the typical temperature $T_{L}$ (15) for the generation of $\Delta L_{\alpha}$ as $T_{L} \gtrsim T_{W}$, and $N_{2}$ and $N_{3}$ are quasi-degenerate, i.e. $\Delta M \ll M_{N}$ where $M_{3}=M_{N}+\Delta M / 2$ and $M_{2}=M_{N}-\Delta M / 2$. 
These are arranged in order to enhance the production of the baryon asymmetry. In this case, we find that the analytical expression of $Y_{B}$ for both the $\mathrm{NH}$ and $\mathrm{IH}$ cases is given by

$$
\begin{aligned}
Y_{B} & =1.8 \times 10^{-11} \kappa\left(T_{L} / T_{W}\right) \frac{M_{0}^{7 / 3} M_{N}^{5 / 3} m_{\mathrm{atm}}^{5 / 2} m_{\mathrm{sol}}^{1 / 2}}{T_{W}\left(\Delta M_{32}^{2} / M_{N}^{2}\right)^{2 / 3}\langle\Phi\rangle^{6}} \times \delta_{\mathrm{CP}} \\
& =4.7 \times 10^{-10} \delta_{\mathrm{CP}}\left(\frac{10^{2} \mathrm{GeV}}{T_{W}}\right)\left(\frac{M_{N}}{5 \mathrm{GeV}}\right)^{5 / 3}\left(\frac{10^{-8}}{\Delta M_{32}^{2} / M_{N}^{2}}\right)^{2 / 3},
\end{aligned}
$$

where we have taken $\kappa\left(T_{L} / T_{W}\right)=1$ in the last equality. The CP asymmetry parameter $\delta_{\mathrm{CP}}$ for BAU is expressed as

$$
\delta_{\mathrm{CP}}=\xi \sin 2 \operatorname{Re} \omega \cdot S_{m_{\nu}} \delta_{\nu}
$$

Here $S_{m_{\nu}}$ is the parameter concerning with the neutrino mass hierarchy in Eq. (20) or (24) for the $\mathrm{NH}$ or IH case, respectively. Therefore, $Y_{B}$ in the IH case is suppressed by about $4 \%$ compared with the NH case apart from $\delta_{\nu}$.

It can be seen that to get the sizable $Y_{B}$ the mass degeneracy of $N_{2}$ and $N_{3}$ at a rather high accuracy is required. Note, however, that such a small mass difference is stable against the radiative corrections due to the smallness of Yukawa coupling constants of neutrinos under consideration. We also find that a larger $Y_{B}$ can be obtained for a larger $M_{N}$ as long as $M_{N} \lesssim 17 \mathrm{GeV}$. Otherwise, $N_{2}$ and $N_{3}$ get in thermal equilibrium and the asymmetries are washed out. It should be noted that $Y_{B}$ is proportional to $\xi \sin \operatorname{Re} \omega$, which means that the sign of $Y_{B}$ cannot be uniquely predicted even when all the parameters of active neutrinos are experimentally determined.

In this expression we have introduced the CP asymmetry parameter $\delta_{\nu}$ to describe how $Y_{B}$ depends on the mixing angles and $\mathrm{CP}$ violating phases in the mixing matrix $U$. The analytical expression for $\delta_{\nu}$ for both the $\mathrm{NH}$ and $\mathrm{IH}$ cases are presented in Appendix A, From now on we shall study these dependence by using the analytical expression as well as the numerical estimation of $Y_{B}$, which is obtained by solving numerically Eqs. (9) and (10).

In the NH case we find that the leading term of $\delta_{\nu}$ in the expansion of $r_{m}$ is given by

$$
\begin{aligned}
\delta_{\nu}= & \frac{1}{2} \sin \theta_{12} \sin 2 \theta_{13}\left[\cos ^{2} \theta_{13}\left(3+\cos 4 \theta_{23}\right)-4 \sin ^{2} \theta_{13}\right] \sin (\delta+\eta) \\
& +\cos \theta_{12} \sin 4 \theta_{23} \cos ^{3} \theta_{13} \sin \eta+\mathcal{O}\left(r_{\mathrm{m}}\right) .
\end{aligned}
$$

It is seen that $\delta_{\nu}$ depends on the $\mathrm{CP}$ violating phases in two ways, i.e., $\delta+\eta$ and $\eta$.

We then find that when the mixing angle in the atmospheric neutrino oscillation is maximal $\theta_{23}=\pi / 4$, the leading term of $\delta_{\nu}$ depends only on the sum of the CP phases $\delta+\eta$. This behaviour can be understood in the left panel of Fig. 2, which represents the parameter region accounting for the present observation data of $Y_{B}$ in the $\delta-\eta$ plane obtained by the numerical solutions of Eqs. (9) and (10). When $\theta_{23}$ is slightly smaller than $\pi / 4$, the allowed region becomes drastically changed as shown in the right panel of Fig. 2. Thus, the deviation of $\theta_{23}$ from $\pi / 4$, 

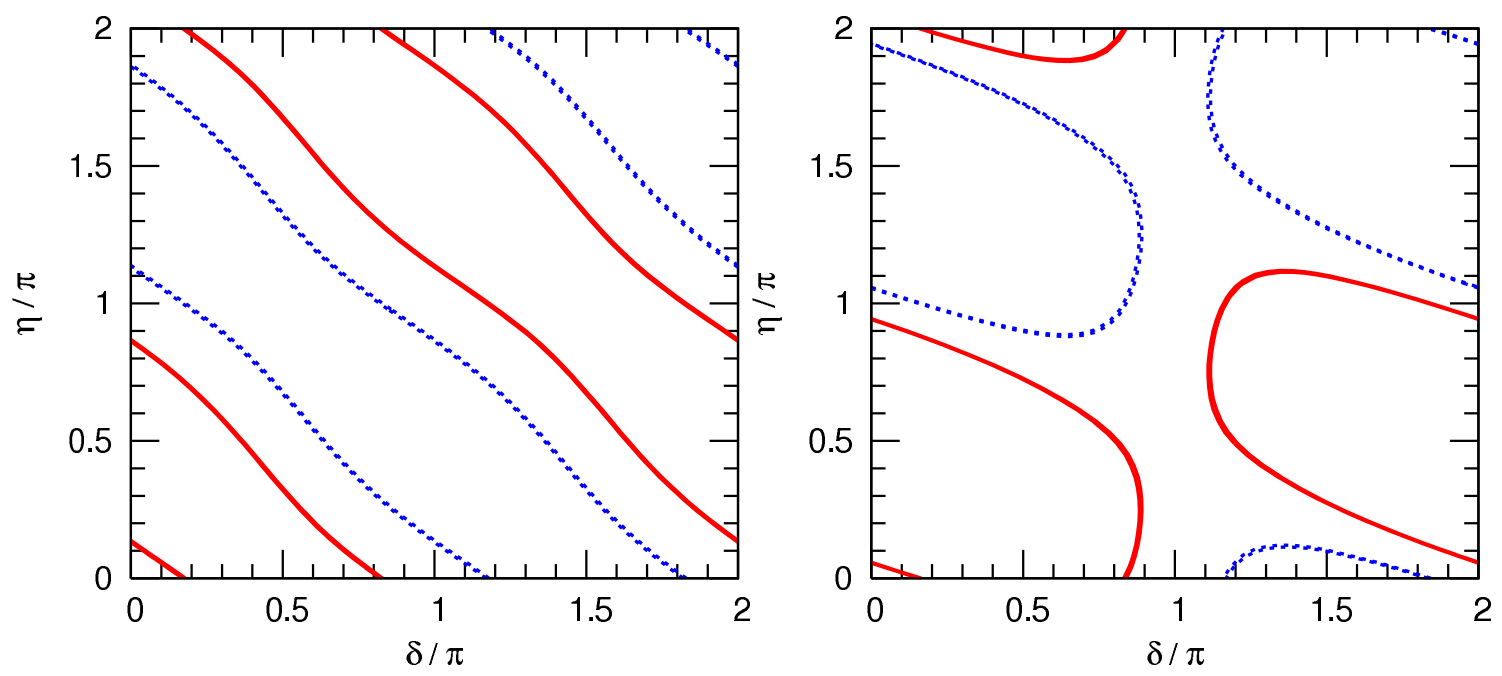

Figure 2: Parameter regions in the $\delta-\eta$ plane accounting for the observed baryon asymmetry in the NH case are shown by lines. The red solid lines are for $\xi=+1$ while the blue dashed lines are for $\xi=-1$. Here we take $M_{3}=5 \mathrm{GeV}, \Delta M_{32}^{2} / M_{3}^{2}=10^{-8}$, Re $\omega=\pi / 4$, and $\sin ^{2} \theta_{13}=0.053$. We take $\sin ^{2} \theta_{23}=0.5$ (left) and 0.36 (right), respectively.

which will be tested in future oscillation experiments, is significant to determine $Y_{B}$ in the considering case.

On the other hand, when $\theta_{13}=0$, the $\mathrm{CP}$ asymmetry parameter becomes

$$
\delta_{\nu}=\sin 4 \theta_{23} \cos \theta_{12}\left(1-r_{m} \cos ^{2} \theta_{12}\right) \sin \eta
$$

including the higher order term of $r_{m}$. In this case the asymmetry depends only on the Majorana phase $\eta$ as expected (since the Dirac phase $\delta$ always appears together with $s_{13}$ ). It is very important to note that $\delta_{\nu}=0$ when $\theta_{13}=0$ and $\theta_{23}=\pi / 4$. In this case, the generation of BAU in the $\mathrm{NH}$ case is ineffective and $Y_{B}$ at $\mathcal{O}\left(F^{6}\right)$ vanishes.

Next, we turn to consider the IH case, where the CP asymmetry parameter $\delta_{\nu}$ at $\mathcal{O}\left(r_{m}^{0}\right)$ is estimated as

$$
\begin{aligned}
\delta_{\nu}= & \frac{1}{4} \sin 2 \theta_{12} \cos ^{2} \theta_{13}\left[-5-3 \cos 4 \theta_{23}+\cos 2 \theta_{13}\left(7+\cos 4 \theta_{23}\right)\right] \sin \eta \\
& +\sin 4 \theta_{23} \cos ^{2} \theta_{13} \sin \theta_{13}\left(\sin \delta \cos \eta-\cos 2 \theta_{12} \cos \delta \sin \eta\right)+\mathcal{O}\left(r_{m}^{2}\right) .
\end{aligned}
$$

It is then found that $\delta_{\nu}$ at the leading order depends only on the Majorana phase when $\theta_{23}=$ $\pi / 4$, which should be compared with the NH case. This behaviour is shown in the left panel of Fig. 3. Moreover, we discover that $\delta_{\nu}$ in the IH case does not vanish even when $\theta_{23}=\pi / 4$ and $\theta_{13}=0$;

$$
\begin{aligned}
\delta_{\nu} & =\frac{1}{2}\left[1+\left(1+r_{m}^{2}\right)^{1 / 2}+3\left[1-\left(1+r_{m}^{2}\right)^{1 / 2}\right] \cos 2 \theta_{12}\right] \sin 2 \theta_{12} \sin \eta \\
& =\sin 2 \theta_{12} \sin \eta\left[1+\frac{1}{4}\left(1-3 \cos 2 \theta_{12}\right) r_{m}^{2}+\mathcal{O}\left(r_{m}^{4}\right)\right] .
\end{aligned}
$$



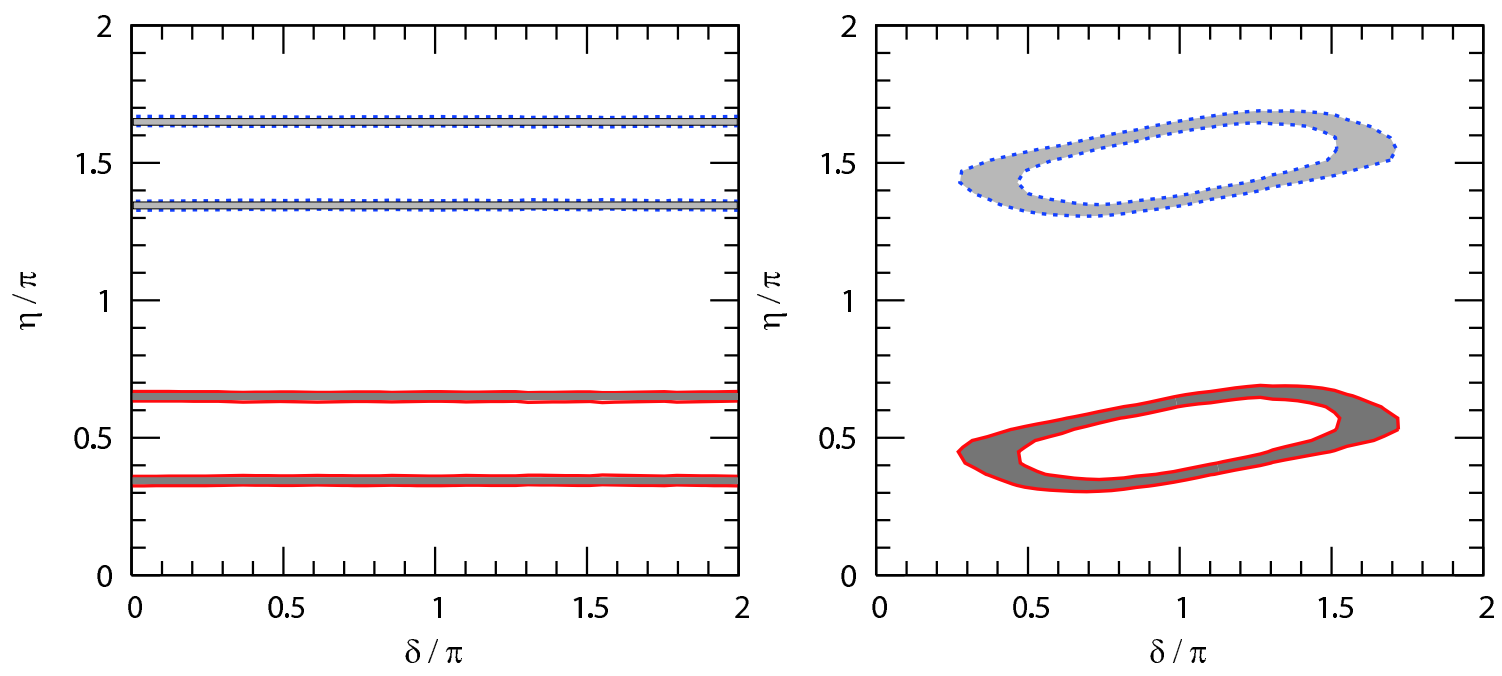

Figure 3: Parameter regions in the $\delta-\eta$ plane accounting for the observed baryon asymmetry in the IH case are shown by shaded regions. The red solid lines are for $\xi=+1$ while the blue dashed lines are for $\xi=-1$. Here we take $M_{3}=15 \mathrm{GeV}, \Delta M_{32}^{2} / M_{3}^{2}=10^{-8}$, Re $\omega=\pi / 4$, and $\sin ^{2} \theta_{13}=0.053$. We take $\sin ^{2} \theta_{23}=0.5$ (left) and 0.36 (right), respectively.

This is one important feature of generating BAU in the IH case.

Therefore, $Y_{B}$ in the IH case is smaller than the NH case in the most of the parameter space, since it receives extra suppression factor of $S_{m_{\nu}}$ in Eq. (24). See Fig. 4. However, when $\theta_{13}$ is very small and also $\theta_{23}$ is almost maximal, baryogenesis in the $\mathrm{NH}$ case becomes ineffective and hence the IH of active neutrino masses is the essence of accounting for the present BAU in the $\nu \mathrm{MSM}$.

\section{Conclusions}

We have studied baryogenesis in the $\nu$ MSM via flavour oscillation between right-handed neutrinos $N_{2}$ and $N_{3}$. In particular, the case when BAU comes solely from the CP violating phases in the mixing matrix of active neutrinos has been investigated. We have presented the analytical expressions of BAU for both the $\mathrm{NH}$ and $\mathrm{IH}$ cases of active neutrino masses, and have demonstrated how the present value of BAU depends on the Dirac and Majorana phases as well as the neutrino mixing angles. We have shown that BAU depends on the neutrino mass hierarchy and $Y_{B}$ in the IH case receives the suppression factor of $S_{m_{\nu}} \simeq 4 \%$. It has been found that $Y_{B}$ is very sensitive to the mixing angles $\theta_{23}$ and $\theta_{13}$. When $\theta_{23}=\pi / 4$, the leading contribution to $Y_{B}$ is proportional to $\sin (\delta+\eta)$ for the $\mathrm{NH}$ case while to $\sin \eta$ for the IH case. Moreover, when $\theta_{23}$ is almost maximal and $\theta_{13}$ is very small, the $\mathrm{CP}$ asymmetry parameter in $Y_{B}$ vanishes and no baryon asymmetry is generated (at least the leading $\mathcal{O}\left(F^{6}\right)$ contribution) in the $\mathrm{NH}$ case. In this case, the IH case is required to explain the observed BAU. 

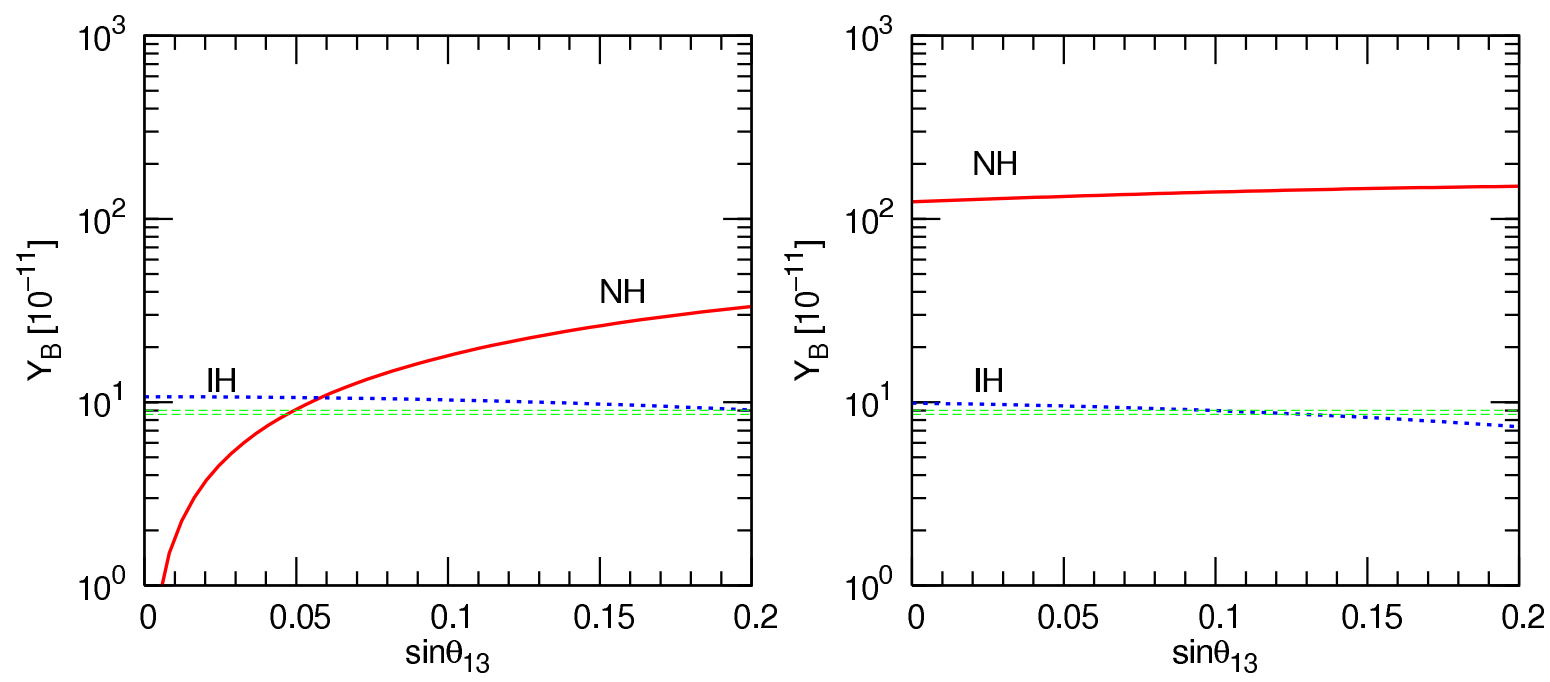

Figure 4: $Y_{B}$ in terms of $\sin \theta_{13}$ are shown by lines. The red solid line is for the NH case while the blue dashed line is for the IH case. The horizontal, green long dashed lines show the region for the observed baryon asymmetry. Here we take $M_{3}=15 \mathrm{GeV}, \Delta M_{32}^{2} / M_{3}^{2}=10^{-8}$, $\operatorname{Re} \omega=\pi / 4, \delta=7 \pi / 4$, and $\eta=\pi / 3$. We take $\sin ^{2} \theta_{23}=0.5$ (left) and 0.36 (right), respectively.

\section{Acknowledgments}

The work of T.A. was partially supported by the Ministry of Education, Science, Sports and Culture, Grant-in-Aid for Scientific Research, No. 21540260, and by Niigata University Grant for Proportion of Project. We thank Mikhail Shaposhnikov for correspondence and reading of the manuscript.

\section{A CP Asymmetry Parameter $\delta_{\nu}$}

In this appendix, we write down the CP asymmetry parameter $\delta_{\nu}$ defined in Eq. (31). This parameter can be found from

$$
\sum_{I=2,3} \sum_{\alpha=e, \mu, \tau}\left|F_{\alpha I}\right|^{2} a_{\alpha}=\frac{m_{\mathrm{atm}} M_{N}}{2\langle\Phi\rangle^{2}} \delta_{\nu}
$$

where we have neglected the terms which are proportional to $\Delta M / M_{N}$.

In the NH case, we can parameterize $\delta_{\nu}$ as

$$
\delta_{\nu}=d_{1}+r_{m} d_{2}
$$

The leading term $d_{1}$ is estimated as

$$
\begin{aligned}
d_{1}= & \frac{1}{2} \sin \theta_{12} \sin 2 \theta_{13}\left[\cos ^{2} \theta_{13}\left(3+\cos 4 \theta_{23}\right)-4 \sin ^{2} \theta_{13}\right] \sin (\delta+\eta) \\
& +\cos \theta_{12} \sin 4 \theta_{23} \cos ^{3} \theta_{13} \sin \eta+\mathcal{O}\left(r_{\mathrm{m}}\right),
\end{aligned}
$$


which had already shown in Eq. (32). For the sub-leading term we can find that

$$
\begin{aligned}
d_{2}= & -2 \sin \theta_{12} \cos ^{2} \theta_{12} \sin 4 \theta_{23} \cos \theta_{13}\left(1+\sin ^{2} \theta_{12} \cos 2 \theta_{13}\right) \sin \eta \\
& -\sin \theta_{12} \cos ^{2} \theta_{12} \sin ^{2} \theta_{23} \sin 2 \theta_{13} \sin (\delta-\eta) \\
& +\frac{1}{8} \sin 2 \theta_{13}\left[-2 \sin ^{3} \theta_{12} \cos 2 \theta_{13}\left(7+\cos 4 \theta_{23}\right)+\sin ^{2} 2 \theta_{23}\left(\sin \theta_{12}+5 \sin 3 \theta_{12}\right)\right] \sin (\delta+\eta) \\
& +2 \sin ^{3} \theta_{12} \cos ^{2} \theta_{12} \sin 4 \theta_{34} \sin ^{2} \theta_{13} \cos \theta_{13} \sin (2 \delta+\eta) .
\end{aligned}
$$

In the IH case, on the other hand, we can parameterize $\delta_{\nu}$ as

$$
\delta_{\nu}=\left(1+r_{m}^{2}\right)^{\frac{1}{2}} d_{1}+d_{2}
$$

where

$$
\begin{aligned}
d_{1}= & {\left[\frac{1}{8} \sin 2 \theta_{12} \cos ^{2} \theta_{13}\left\{-5-3 \cos 4 \theta_{23}+\cos 2 \theta_{13}\left(7+\cos 4 \theta_{23}\right)\right\}\right.} \\
& \left.-\frac{1}{64} \sin 4 \theta_{12}\left\{29+27 \cos 4 \theta_{23}+\cos 4 \theta_{13}\left(7+\cos 4 \theta_{23}\right)+40 \sin ^{2} \theta_{23} \cos 2 \theta_{13}\right\}\right] \sin \eta \\
& +\frac{1}{8} \sin 4 \theta_{23}\left[4 \sin \theta_{13}\left\{\cos ^{2} \theta_{13}+\cos \theta_{12}\left(1+\sin ^{2} \theta_{13}\right)\right\} \sin \delta \cos \eta\right. \\
& \left.-\left\{4 \cos 2 \theta_{12} \sin \theta_{13} \cos ^{2} \theta_{13}+\cos 4 \theta_{12}\left(7 \sin \theta_{13}-\sin 3 \theta_{13}\right)\right\} \cos \delta \sin \eta\right] \\
& -\frac{1}{2} \sin ^{2} 2 \theta_{23} \sin ^{2} \theta_{13}\left(2 \sin 2 \theta_{12} \sin 2 \delta \cos \eta-\sin 4 \theta_{12} \cos 2 \delta \sin \eta\right), \\
d_{2}= & \frac{1}{8} \sin 2 \theta_{12} \cos ^{2} \theta_{13}\left\{-5-3 \cos 4 \theta_{23}+\cos 2 \theta_{13}\left(7+\cos 4 \theta_{23}\right)\right\} \\
& \left.+\frac{1}{64} \sin 4 \theta_{12}\left\{29+27 \cos 4 \theta_{23}+\cos 4 \theta_{13}\left(7+\cos 4 \theta_{23}\right)+40 \sin \theta_{23} \cos 2 \theta_{13}\right\}\right] \sin \eta \\
& +\frac{1}{8} \sin 4 \theta_{23}\left[\left\{\sin \theta_{13}\left(1-7 \cos 2 \theta_{12}\right)+2 \cos ^{2} \theta_{12} \sin 3 \theta_{13}\right\} \sin \delta \cos \eta\right. \\
& \left.-\left\{4 \cos 2 \theta_{12} \sin \theta_{13} \cos ^{2} \theta_{13}+\cos 4 \theta_{12}\left(-7 \sin \theta_{13}+\sin 3 \theta_{13}\right)\right\} \cos \delta \sin \eta\right] \\
& +\frac{1}{4} \sin \theta_{13}\left[\sin 2 \theta_{12} \sin ^{2} 2 \theta_{23} \sin 2 \delta \cos \eta-\sin 4 \theta_{23}\left(1-\cos 4 \theta_{23}\right) \cos 2 \delta \sin \eta\right] .
\end{aligned}
$$

Actually, the leading term of Eq. (40), i.e., $\left.\delta_{\nu}\right|_{r_{m}=0}$ is obtained from $d_{1}+d_{2}$ as,

$$
\begin{aligned}
d_{1}+d_{2}= & \frac{1}{4} \sin 2 \theta_{12} \cos ^{2} \theta_{13}\left[\left\{-5-3 \cos 4 \theta_{23}+\cos 2 \theta_{13}\left(7+\cos 4 \theta_{23}\right)\right\} \sin \eta\right. \\
& \left.+4 \sin \theta_{13} \sin 4 \theta_{23}\left(\sin \delta \cos \eta-\cos 2 \theta_{12} \cos \delta \sin \eta\right)\right] .
\end{aligned}
$$

This conclusion is consistent with Eq. (34).

\section{References}

[1] A. Riotto and M. Trodden, Ann. Rev. Nucl. Part. Sci. 49 (1999) 35 arXiv:hep-ph/9901362. 
[2] M. Fukugita and T. Yanagida, Phys. Lett. B 174 (1986) 45.

[3] W. Buchmuller, R. D. Peccei and T. Yanagida, Ann. Rev. Nucl. Part. Sci. 55 (2005) 311 arXiv:hep-ph/0502169.

[4] P. Minkowski, Phys. Lett. B 67 (1977) 421; T. Yanagida, in Proc. of the Workshop on the Unified Theory and the Baryon Number in the Universe, Tsukuba, Japan, Feb. 13-14, 1979, p. 95, eds. O. Sawada and S. Sugamoto, (KEK Report KEK-79-18, 1979, Tsukuba); Progr. Theor. Phys. 64 (1980) 1103 ; M. Gell-Mann, P. Ramond and R. Slansky, in Supergravity, eds. P. van Niewenhuizen and D. Z. Freedman (North Holland, Amsterdam 1980); P. Ramond, in Talk given at the Sanibel Symposium, Palm Coast, Fla., Feb. 25Mar. 2, 1979, preprint CALT-68-709 (retroprinted as hep-ph/9809459); S. L. Glashow, in Proc. of the Cargése Summer Institute on Quarks and Leptons, Cargése, July 9-29, 1979, eds. M. Lévy et. al, , (Plenum, 1980, New York), p707.

[5] G. F. Giudice, A. Notari, M. Raidal, A. Riotto and A. Strumia, Nucl. Phys. B 685 (2004) 89 arXiv:hep-ph/0310123.

[6] T. Asaka and M. Shaposhnikov, Phys. Lett. B 620 (2005) 17.

[7] T. Asaka, S. Blanchet and M. Shaposhnikov, Phys. Lett. B 631 (2005) 151.

[8] D. Gorbunov and M. Shaposhnikov, JHEP 0710 (2007) 015 [arXiv:0705.1729 [hep-ph]].

[9] E. K. Akhmedov, V. A. Rubakov and A. Y. Smirnov, Phys. Rev. Lett. 81 (1998) 1359.

[10] M. Shaposhnikov, JHEP 0808 (2008) 008 [arXiv:0804.4542 [hep-ph]].

[11] V. A. Kuzmin, V. A. Rubakov and M. E. Shaposhnikov, Phys. Lett. B 155 (1985) 36.

[12] T. Asaka, M. Laine and M. Shaposhnikov, JHEP 0701 (2007) 091 arXiv:hep-ph/0612182.

[13] M. Laine and M. Shaposhnikov, JCAP 0806 (2008) 031 [arXiv:0804.4543 [hep-ph]].

[14] A. Boyarsky, O. Ruchayskiy and M. Shaposhnikov, Ann. Rev. Nucl. Part. Sci. 59 (2009) 191 [arXiv:0901.0011 [hep-ph]].

[15] J. A. Casas and A. Ibarra, Nucl. Phys. B 618 (2001) 171 arXiv:hep-ph/0103065.

[16] For example, see, T. Schwetz, M. A. Tortola and J. W. F. Valle, New J. Phys. 10 (2008) 113011 arXiv:0808.2016 [hep-ph]].

[17] T. Asaka and H. Ishida, in preparation.

[18] S. Y. Khlebnikov and M. E. Shaposhnikov, Nucl. Phys. B 308 (1988) 885; J. A. Harvey and M. S. Turner, Phys. Rev. D 42 (1990) 3344. 